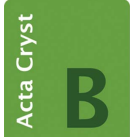

STRUCTURAL SCIENCE CRYSTAL ENGINEERING MATERIALS

ISSN 2052-5206

Received 18 May 2015

Accepted 18 May 2015

Keywords: high-pressure crystallography; dense framework materials; magnetism; coordination polymers.

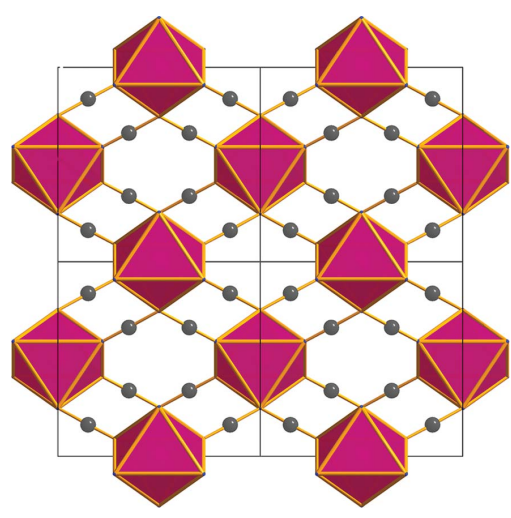

C 2015 International Union of Crystallography

\section{Probing the structure of framework materials by high pressure and the example of a magnetic, non- porous coordination polymer}

\author{
Francesca P. A. Fabbiani* \\ Georg-August-Universität Göttingen, GZG, Abteilung Kristallographie, Goldschmidtstrasse 1, 37077 Göttingen, \\ Germany. ${ }^{*}$ Correspondence e-mail: ffabbia@gwdg.de
}

Nestled between the modest pressures employed to study protein folding (a few hundred megapascals) and the very high pressures obtained in shock-wave experiments (in the order of terapascals), a vast pressure scale can be explored experimentally and computationally in the physical and life sciences. Pressure is a powerful thermodynamic variable that enables the structure, bonding and reactivity of matter to be altered. In materials science it has become an indispensable research tool in the quest for novel functional materials. Examples include the target synthesis of new classes of materials with unique physical properties, see for instance the recently reported ultra-thin 'diamond nanothreads' obtained by decompressing benzene from $20 \mathrm{GPa}$ to ambient pressure (Fitzgibbons et al., 2015). Materials scientists can exploit the effectiveness of pressure for probing and tuning structural, mechanical, electronic, magnetic and vibrational properties of materials in situ; crystallography plays a crucial role here, enabling on one hand the unravelling of structural phenomena through a better understanding of interactions, and on the other hand the derivation of structure-property relationships.

Crystalline framework materials have long established themselves as the subject of intense interdisciplinary research activity; several classification systems and topological descriptors exist and the terminology found in the literature is rich and varied. In the simple and somewhat arbitrary view of this author, these materials can be divided into two main classes based on property, namely materials that exhibit porosity and those that do not. Porosity allows for potential applications in, inter alia, energy storage, catalysis, separation and capture technologies. Examples of industrially relevant framework materials displaying porosity include zeolites, at the forefront of the family of inorganic open-framework materials (Cheetham et al., 1999), and metal-organic frameworks (MOFs), which in the literature appear under the heading of hybrid inorganic organic framework materials (Cheetham et al., 2006) and, following the preferred definition by IUPAC (Batten et al., 2012, 2013), under the heading of coordination networks, a subclass of coordination polymers. In the past decade MOFs have sparked an international frenzy of research activity. Porous framework materials have been widely investigated by highpressure crystallography: zeolites have been recently reviewed (Gatta \& Lee, 2014) and a review on the subject of 'MOFs under high pressure' by S. A. Moggach will appear later in 2015 as a feature article in Acta Crystallographic Section B. In a very recent perspective in Chemistry of Materials, Coudert (2015) provides an inspiring overview of porous and non-porous 'stimuli-responsive' framework materials, i.e. those framework materials that by virtue of their flexibility undergo marked structural changes ('changes of large amplitude') in response to external stimuli such as pressure, temperature or light.

High-pressure structural investigations of dense, i.e. non-porous, framework materials based on coordination compounds have received somewhat less attention compared with their porous counterparts. However, with pressure promoting effects such as magnetic crossover, spin transitions, negative linear compressibility (NLC; for a very recent and elegant review on this subject see Cairns \& Goodwin, 2015), changes in proton conductivity, or even phase transitions that generate porous structures, high-pressure crystallographic studies on dense framework materials are markedly on the rise. More generally, coordination compounds are a fascinating class of materials for high-pressure crystallographic studies (Tidey et al., 2014; Moggach \& Parsons, 2009). Compared with purely organic compounds, they have an inherent extra degree of flexibility for 
responding to moderate applied pressures $(<5 \mathrm{GPa})$, as the geometry at the metal centre can undergo marked changes, whereas other primary bond distances and angles remain largely unaffected.

Among framework materials, coordination polymers containing pseudohalide ligands have attracted considerable interest for their versatility of coordination modes, giving rise to a wide range of porous and non-porous structures that can exhibit interesting magnetic properties at ambient-pressure conditions (Batten et al., 2009). In this context, the small polynitrile dicyanamide (dca) and tricyanomethanide (tcm) ligands have been reviewed by Batten \& Murray (2003). These particular systems have just started to catch the attention of the high-pressure structural community. Non-magnetic $\mathrm{Ag}(\mathrm{tcm})$ was recently reported to exhibit the rare and technologically interesting phenomenon of negative area compressibility (Hodgson et al., 2014). Yakovenko et al. (2015) are the first to report a high-pressure crystallographic study on a magnetic coordination polymer containing the dca ligand. The authors elucidate the structure of a high-pressure $\gamma$-phase of $\mathrm{Co}(\mathrm{dca})_{2}$, a molecule-based magnet, obtained by compression of a polycrystalline sample of the $\alpha$-form to $1.1 \mathrm{GPa}$ at ambient temperature (Fig. 1). The study by Yakovenko et al. (2015) beautifully illustrates several of the concepts enumerated above. $\alpha$-Co(dca $)_{2}$, a collinear ferromagnet with $T_{\mathrm{c}}=9 \mathrm{~K}$, is a dense framework material with rutile-like topology. As the $\alpha$-form is compressed, $\mathrm{Co}^{\mathrm{II}}$ octahedra rotate and are substantially elongated along the axial direction, reaching a maximum value well in excess of typical lengths observed for coordinative $\mathrm{Co}-\mathrm{N}$ bonds. The amide $\mathrm{C}-\mathrm{N}-\mathrm{C}$ bond angle becomes increasingly strained, surging to values in excess of $150^{\circ}$ just before the transition pressure, an increase of over $30^{\circ}$ compared with the ambient-pressure value. Immediately after the transition, which is reversible, the axial distortion is considerably reduced, but the aforementioned strain is retained. These substantial structural changes are all the more remarkable when considering the very modest pressures required to induce the phase transition and testify to the material's structural flexibility. Both $\alpha$ - and $\gamma$-Co(dca $)_{2}$ exhibit modest NLC through a 'wine-rack' mechanism. The high-pressure magnetic behaviour of $\alpha$-Co(dca $)_{2}$ had been previously investigated by Nuttall et al. (2000), who reported a pressure-induced transformation to antiferromagnetic behaviour at $c a 1 \mathrm{GPa}$. While the derivation of widely applicable theories for explaining structure-property relationships are particularly appealing and ultimately needed, Yakovenko et al. (2015) are rightly careful about drawing conclusions on structure-magnetic correlations at high pressure. Although it is generally accepted that long-range magnetic coupling in $\alpha$ $M(\mathrm{dca})_{2}$ materials occurs via superexchange and more precisely through interaction through the $\mathrm{N}-\mathrm{C} \equiv \mathrm{N}$ units, a detailed ambient-pressure low-temperature neutron study (Lappas et al., 2003) has shown that structural parameters alone are not reliable indicators for predicting the magnetic ground state. In fact, the origin of the magnetic behaviour at ambient pressure is still not fully understood. Further discussion of high-pressure magnetism would require complementary studies to determine the nuclear and magnetic structures at high pressure and at temperatures below and above the onset of magnetic ordering.

A large number of magnetic and non-magnetic coordination polymers incorporating the dca ligand in its various coordination modes have already been structurally characterized at ambient pressure and many more are in principle waiting to be studied or yet synthesized. Some materials, including $\mathrm{Co}(\mathrm{dca})_{2}$, are polymorphic and exhibit different coordination numbers and magnetic ground states; in theory, by choosing an appropriate starting material, a transition to an arrangement in which a complete structural reorganization takes place with the metal adopting a higher coordination number may even be possible at sufficiently high pressures. The high-pressure crystallographic study of Yakovenko et al. (2015) advocates that a wider range of related samples be studied under high-pressure conditions and paves the way for a series of future investigations employing a combination of different techniques for material characterization that could help improve our understanding of structure-property correlations in related non-porous structures, thereby contributing to the design of the next generation of pressureresponsive, functional materials.
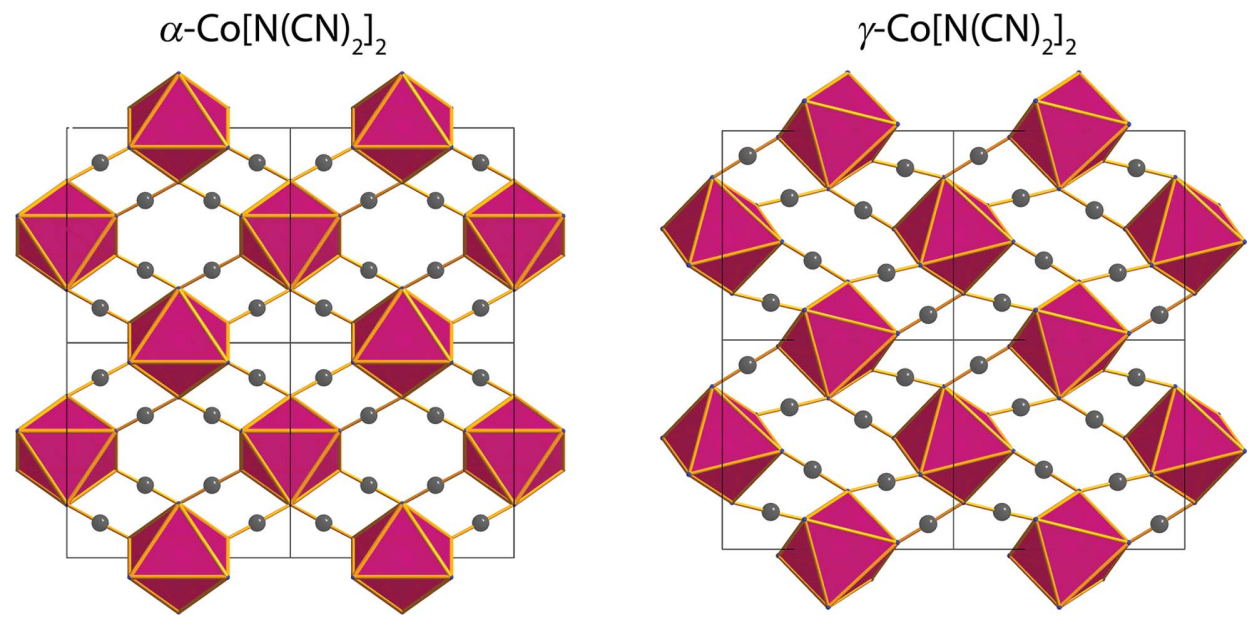

Figure 1

$\mathrm{Co}^{\mathrm{II}}$ octahedral packing diagrams viewed in the (001) plane, reprinted with permission from Yakovenko et al. (2015). 


\section{scientific commentaries}

S., Öhrström, L., O'Keeffe, M., Suh, M. P. \& Reedijk, J. (2013). Pure Appl. Chem. 85, 1715-1724.

Batten, S. R. \& Murray, K. S. (2003). Coord. Chem. Rev. 246, 103-130.

Batten, S. R., Turner, D. R. \& Neville, M. S. (2009). Coordination Polymers: Design, Analysis and Application. Cambridge, UK: Royal Society of Chemistry.

Cairns, A. B. \& Goodwin, A. L. (2015). ArXiv: 1502.00846.

Cheetham, A. K., Férey, G. \& Loiseau, T. (1999). Angew. Chem. Int. Ed. 38, 3268-3292.

Cheetham, A. K., Rao, C. N. R. \& Feller, R. K. (2006). Chem. Commun. p. 4780.

Coudert, F.-X. (2015). Chem. Mater. 27, 1905-1916.

Fitzgibbons, T. C., Guthrie, M., Xu, E., Crespi, V. H., Davidowski, S. K., Cody, G. D., Alem, N. \& Badding, J. V. (2015). Nat. Mater. 14, 43-47.
Gatta, G. D. \& Lee, Y. (2014). Mineral. Mag. 78, 267291.

Hodgson, S. A., Adamson, J., Hunt, S. J., Cliffe, M. J., Cairns, A. B., Thompson, A. L., Tucker, M. G., Funnell, N. P. \& Goodwin, A. L. (2014). Chem. Commun. 50, 5264-5266.

Lappas, A., Wills, A. S., Green, M. A., Prassides, K. \& Kurmoo, M. (2003). Phys. Rev. B, 67, 144406.

Moggach, S. A. \& Parsons, S. (2009). Spectrosc. Prop. Inorg. Organomet. Compd. 40, 324-354.

Nuttall, C. J., Takenobu, T., Iwasa, Y. \& Kurmoo, M. (2000). Mol. Cryst. Liq. Cryst. Sci. Technol. Sect. A, 343, 227-234.

Tidey, J. P., Wong, H. L. S., Schröder, M. \& Blake, A. J. (2014). Coord. Chem. Rev. 277-278, 187-207.

Yakovenko, A., Chapman, K. \& Halder, G. (2015). Acta Cryst. B71, 252-257. 